\title{
Average and extreme multi-atom Van der Waals interactions: Strong coupling of multi-atom Van der Waals interactions with covalent bonding
} Alexei V Finkelstein

Address: Institute of Protein Research, Russian Academy of Sciences,142290, Pushchino, Moscow Region, Russia

Email: Alexei V Finkelstein - afinkel@vega.protres.ru

Published: 30 July 2007

Chemistry Central Journal 2007, I:21 doi:I0.1 |86/1752-153X-I-2I
Received: 30 March 2007

Accepted: 30 July 2007

This article is available from: http://journal.chemistrycentral.com/content/I/I/2I

(C) 2007 Finkelstein et al

This is an Open Access article distributed under the terms of the Creative Commons Attribution License (http://creativecommons.org/licenses/by/2.0), which permits unrestricted use, distribution, and reproduction in any medium, provided the original work is properly cited.

\begin{abstract}
Background: The prediction of ligand binding or protein structure requires very accurate force field potentials - even small errors in force field potentials can make a 'wrong' structure (from the billions possible) more stable than the single, 'correct' one. However, despite huge efforts to optimize them, currently-used all-atom force fields are still not able, in a vast majority of cases, even to keep a protein molecule in its native conformation in the course of molecular dynamics simulations or to bring an approximate, homology-based model of protein structure closer to its native conformation.
\end{abstract}

Results: A strict analysis shows that a specific coupling of multi-atom Van der Waals interactions with covalent bonding can, in extreme cases, increase (or decrease) the interaction energy by about $20-40 \%$ at certain angles between the direction of interaction and the covalent bond. It is also shown that on average multi-body effects decrease the total Van der Waals energy in proportion to the square root of the electronic component of dielectric permittivity corresponding to dipoledipole interactions at small distances, where Van der Waals interactions take place.

Conclusion: The study shows that currently-ignored multi-atom Van der Waals interactions can, in certain instances, lead to significant energy effects, comparable to those caused by the replacement of atoms (for instance, $\mathrm{C}$ by $\mathrm{N}$ ) in conventional pairwise Van der Waals interactions.

\section{Background}

Van der Waals (VdW) forces, which are very important for the structure and interactions of biological molecules, are usually treated as a simple sum of pairwise inter-atomic interactions even in dense systems like proteins [1-5]. However, multi-atom VdW interactions are usually ignored. This seems to follow the Axilrod-Teller theory [6] which predicts a drastic (stronger than for pairwise interactions) decrease of three-atom interactions with distance; and indeed, detailed computations of single-atom liquids [7] and solids [8,9] show that MB (multi-body) effects amount to only $\sim 5 \%$ of the total energy. However, this work shows that multi-atom VdW interactions can become quite large in the presence of covalent bonds. This finding, which equally concerns atomic interactions in biological molecules and solvents, implies a necessity to revise the all-atom force fields currently used.

Results and Discussion

Theory

Following earlier studies $[6,7,10-12]$, each atom $i(i=1,2$, $\ldots, n)$ is considered as a three-dimensional (3D) harmonic 
quantum oscillator, where an electron with mass $m_{i}$ oscillates harmonically with a frequency $\omega_{i}$, if unaffected by other atoms. Atom $i$ is fixed in the 3D point $\mathrm{r}_{i}$; it has an instantaneous dipole moment $e \mathrm{x}_{i}$, where $e$ is the elementary charge and $x_{i}$ the $3 \mathrm{D}$ vector of instantaneous displacement (whose equilibrium value is zero). Thus, a classic Hamiltonian for the system of coupled oscillators is

$$
H=\sum_{i} \frac{m_{i}}{2}\left(\frac{d \mathrm{x}_{i}}{d t}\right)^{2}+\sum_{i} \frac{m_{i} \omega_{i}^{2}}{2} \mathrm{x}_{i}^{2}+\sum_{i} \sum_{j>i} e^{2} \frac{\boldsymbol{\theta}_{i j}}{\left|\mathrm{r}_{i j}\right|^{3}} \mathrm{x}_{i} \mathrm{x}_{j} ;
$$

here $\theta_{i j} /\left|r_{i j}\right|^{3}=\left(\delta-3 n_{i j} \otimes n_{i j}\right) /\left|r_{i j}\right|^{3}$ is the usual dipoledipole interaction tensor (where $\delta$ is the $3 \mathrm{D}$ unit matrix $\mathrm{n}_{i j} \otimes \mathrm{n}_{i j}$ the tensor product of vectors $\mathrm{n}_{i j}=\mathrm{r}_{i j} /\left|\mathrm{r}_{i j}\right|$, and $r_{i j}=$ $\mathrm{r}_{i}-\mathrm{r}_{j} ; \neq j$ ). As is usually done (see Refs. [6,10-12]), relatively week quadruple-dipole and so on interactions of oscillators are ignored in addition to possible inharmoniousness of the oscillators.

\section{Energy of multi-body VdW interactions}

Using the substitution $\mathrm{y}_{i}=\mathrm{x}_{i} \sqrt{m_{i}}$ [12], a conventional quantum mechanical Hamiltonian operator for coupled oscillators is obtained from [1]:

$$
\hat{H}=-\frac{\hbar^{2}}{2} \sum_{i} \frac{\partial^{2}}{\partial \mathrm{y}_{i}^{2}}+\sum_{i} \frac{\omega_{i}^{2}}{2} \mathrm{y}_{i}^{2}+\sum_{i} \sum_{j>i} \frac{e^{2}}{\sqrt{m_{i} m_{j}}} \frac{\boldsymbol{\theta}_{i j}}{\left|\mathrm{r}_{i j}\right|^{3}} \mathrm{y}_{i} \mathrm{y}_{j}
$$

here $\hbar$ is the reduced Planck constant. By the introduction of a $3 n$-dimensional vector $\mathrm{Y}=\left[\mathrm{y}_{1}, \ldots, \mathrm{y}_{n}\right]$ composed of $n 3 \mathrm{D}$ vectors $\mathrm{y}_{i}$, and a $3 n \times 3 n$ matrix $\mathbf{B}=\| \mathbf{b}_{i j}||$ composed of $n \times n \quad 3 \mathrm{D}$ blocks $\mathbf{b}_{i j}\left[\mathbf{b}_{i j}=\omega_{i}^{2} \boldsymbol{\delta}\right.$ if $i=j, \mathbf{b}_{i j}=\left(e^{2} / \sqrt{m_{i} m_{j}}\right)\left(\boldsymbol{\theta}_{i j} /\left|\mathbf{r}_{i j}\right|^{3}\right)$ if $\left.i \neq j\right]$ , we obtain a simple Hamiltonian

$$
\hat{H}=-\frac{\hbar^{2}}{2} \frac{d^{2}}{d \mathrm{Y}^{2}}+\frac{1}{2} \mathrm{YBY}
$$

for oscillation in a $3 n$ dimensional potential well determined by the $3 n \times 3 n$ matrix $\mathbf{B}$. The $3 n$ eigenvalues $\left(\Omega_{1}\right)^{2}$, ..., $\left(\Omega_{3 n}\right)^{2}$ of matrix $\mathbf{B}$ are squared frequencies of $3 n$ independent one-dimensional oscillations along its eigenvectors. If dipole-dipole interactions are absent (i.e., all $\theta_{i j} \equiv$ $\mathbf{0}$, where $\mathbf{0}$ is the zero 3D matrix), $\Omega_{1}=\Omega_{2}=\Omega_{3}=\omega_{1}, \ldots$, $\Omega_{3 n-2}=\Omega_{3 n-1}=\Omega_{3 n}=\Omega_{n}$. If dipole-dipole interactions are small, $\Omega_{1}>0, \ldots, \Omega_{3 n}>0$ are eigenvalues of the positively determined matrix $\mathbf{B}^{1 / 2}$ (while in the case of too large dipole-dipole interactions the system becomes unstable, and at least one $\Omega^{2}$ becomes negative). The frequencies $\Omega$ $>0$ of the stable system of oscillators determine the ground state energy of this system [12]: the system's energy is $W=\sum_{s=1}^{3 n} \frac{\hbar}{2}{ }^{\prime}{ }_{s}=\frac{\hbar}{2} \operatorname{Sp}\left[\mathbf{B}^{1 / 2}\right]$ in the presence of dipole-dipole interactions, while the energy of the same but non-interacting oscillators is $W_{0}=\frac{\hbar}{2} \sum_{i=1}^{n} 3 \omega_{i}$. Thus, the energy of $\mathrm{VdW}$ dispersion forces is

$$
\Delta W=W-W_{0}=\frac{\hbar}{2}\left(\operatorname{Sp}\left[\mathbf{B}^{1 / 2}\right]-\sum_{i=1}^{n} 3 \omega_{i}\right)
$$

In general, one can compute all $3 n$ eigenvalues $\left(\Omega_{i}\right)^{2}$ of $3 n$ $\times 3 n$ matrix $\mathbf{B}$ (and thus eigenvalues $\Omega_{i}$ of $\mathbf{B}^{1 / 2}$ and their sum Sp $\left.\left[\mathbf{B}^{1 / 2}\right]\right)$ in a time proportional to $(3 n)^{3}$. Computationally, this solves a problem of exact calculation of $\mathrm{VdW}$ dispersion forces for any system of polarizable dipoles.

However, to get a physical understanding of the main terms contributing to these forces, one has to consider the main terms of $\mathrm{Sp}\left[\mathrm{B}^{1 / 2}\right]$.

Matrix B can be presented as

$$
\mathbf{B}=\mathbf{B}_{0}+\Delta \mathbf{B}=\left[\begin{array}{cccc}
\omega_{1}^{2} \boldsymbol{\delta} & \mathbf{0} & \ldots & \mathbf{0} \\
\mathbf{0} & \omega_{2}^{2} \boldsymbol{\delta} & \ldots & \mathbf{0} \\
\ldots & \ldots & \ldots & \ldots \\
\mathbf{0} & \mathbf{0} & \ldots & \omega_{n}^{2} \boldsymbol{\delta}
\end{array}\right]+\left[\begin{array}{cccc}
\mathbf{0} & \mathbf{b}_{12} & \ldots & \mathbf{b}_{1 n} \\
\mathbf{b}_{21} & \mathbf{0} & \ldots & \mathbf{b}_{2 n} \\
\ldots & \ldots & \ldots & \ldots \\
\mathbf{b}_{n 1} & \mathbf{b}_{n 2} & \ldots & \mathbf{0}
\end{array}\right]
$$

where matrix $\mathbf{B}_{0}$ corresponds to uncoupled oscillators and $\Delta \mathbf{B}$ to weak coupling of the oscillators. Now let us consider an auxiliary matrix $\mathbf{B} \lambda=\mathbf{B}_{0}+\lambda \Delta \mathbf{B}$ (where $\lambda$ is a small multiplier), and present its square root, $\mathbf{B}_{\lambda}^{1 / 2}$, as a series $\mathbf{B}_{\lambda}^{1 / 2}=\mathbf{B}_{0}^{1 / 2}+\lambda \mathbf{Z}_{1}+\lambda^{2} \mathbf{Z}_{2}+\ldots$, where $\mathbf{B}_{0}^{1 / 2}$ is a diagonal matrix with 3D blocks $\left(\mathbf{B}_{0}^{1 / 2}\right)_{i j}=\omega_{i} \delta$ if $i=j$, and $\left(\mathbf{B}_{0}^{1 / 2}\right)_{i j}$ $=\mathbf{0}$ if $i \neq j$, and matrices $\mathbf{Z}_{1}, \mathbf{Z}_{2}, \ldots$ have to be calculated.

Since

$\mathbf{B}_{\lambda}^{1 / 2} \mathbf{B}_{\lambda}^{1 / 2}=\mathbf{B}_{0}+\lambda\left[\mathbf{B}_{0}^{1 / 2} \mathbf{Z}_{1}+\mathbf{Z}_{1} \mathbf{B}_{0}^{1 / 2}\right]+\lambda^{2}\left[\mathbf{B}_{0}^{1 / 2} \mathbf{Z}_{2}+\mathbf{Z}_{1} \mathbf{Z}_{1}+\mathbf{Z}_{2} \mathbf{B}_{0}^{1 / 2}\right]+\ldots$ and $\mathbf{B}_{\lambda}^{1 / 2} \mathbf{B}_{\lambda}^{1 / 2}=\mathbf{B}_{0}+\lambda \Delta \mathbf{B}$, 
$\mathbf{B}_{0}^{1 / 2} \mathbf{Z}_{1}+\mathbf{Z}_{1} \mathbf{B}_{0}^{1 / 2}=\Delta \mathbf{B}$

$\mathbf{B}_{0}^{1 / 2} \mathbf{Z}_{2}+\mathbf{Z}_{2} \mathbf{B}_{0}^{1 / 2}=-\mathbf{Z}_{1} \mathbf{Z}_{1}$

...

$\mathbf{B}_{0}^{1 / 2} \mathbf{Z}_{m}+\mathbf{Z}_{m} \mathbf{B}_{0}^{1 / 2}=-\left(\mathbf{Z}_{1} \mathbf{Z}_{m-1}+\ldots+\mathbf{Z}_{m-1} \mathbf{Z}_{1}\right) ;$ and so on.

This system can be solved recursively: equation $\mathrm{MZ}+\mathrm{ZM}$ $=\mathbf{Y}$ (where matrices consist of equal-size blocks $(\mathbf{M})_{i j}$ $\left.(\mathrm{Z})_{i j^{\prime}}(\mathrm{Y})_{i j^{\prime}}, i, j=1, \ldots, n\right)$ unambiguously determines $\mathrm{Z}$ for any $\mathbf{Y}$ when $\mathbf{M}=|| \omega_{i} \delta_{i j} \delta \|$ is a positively determined diagonal

matrix:

$(\mathbf{M Z}+\mathbf{Z M})_{i j}=\sum_{p}\left[\omega_{i} \delta_{i p} \boldsymbol{\delta} \cdot(\mathbf{Z})_{p j}+(\mathbf{Z})_{i p} \cdot \omega_{p} \delta_{p j} \boldsymbol{\delta}\right]=\left[\omega_{i}+\omega_{j}\right](\mathbf{Z})_{i j}$

, and from $\left[\omega_{i}+\omega_{j}\right](\mathbf{Z})_{i j}=(\mathbf{Y})_{i j}=(\mathbf{Y})_{i j}$ we have $(Z)_{i j}=(\mathbf{Y})_{i j} /$ $\left(\omega_{i}+\omega_{j}\right)$.

Denoting $\quad 1 /\left(\omega_{i}+\omega_{j}\right)$ as $\mu_{i j}$ and $\mathbf{b}_{i j} /\left(\omega_{i}+\omega_{j}\right)=\left\{e^{2} /\left[\sqrt{m_{i} m_{j}}\left(\omega_{i}+\omega_{j}\right)\right]\right\} \cdot\left(\boldsymbol{\theta}_{i j} /\left|\mathrm{r}_{i j}\right|^{3}\right)$

as $\mathbf{h}_{i j}$, we have

$\left(\mathbf{Z}_{1}\right)_{i j}=(\Delta \mathbf{B})_{i j} /\left(\omega_{i}+\omega_{j}\right)=\mathbf{h}_{i j} ;$

$\left(\mathbf{Z}_{2}\right)_{i j}=-\left(\mathbf{Z}_{1} \mathbf{Z}_{1}\right)_{i j} /\left(\omega_{i}+\omega_{j}\right)=-\sum_{k} \mathbf{h}_{i k} \mathbf{h}_{k j} \cdot \mu_{i j}$;

$\left(\mathbf{Z}_{3}\right)_{i j}=-\left(\mathbf{Z}_{1} \mathbf{Z}_{2}+\mathbf{Z}_{2} \mathbf{Z}_{1}\right)_{i j} /\left(\omega_{i}+\omega_{j}\right)=+\sum_{k} \sum_{p} \mathbf{h}_{i k} \mathbf{h}_{k p} \mathbf{h}_{p j} \cdot\left(\mu_{i p}+\mu_{k j}\right) \mu_{i j} ;$

$\left(\mathbf{Z}_{4}\right)_{i j}=-\sum_{k} \sum_{p} \sum_{q} \mathbf{h}_{i k} \mathbf{h}_{k p} \mathbf{h}_{p q} \mathbf{h}_{q j} \cdot\left(\mu_{i p} \mu_{i q}+\mu_{i q} \mu_{k q}+\mu_{i p} \mu_{p j}+\mu_{k j} \mu_{k q}+\mu_{k j} \mu_{p j}\right) \mu_{i j}$; and so on.

Matrices $Z_{1}, Z_{2}, Z_{3}, \ldots$ do not depend on $\lambda$. Series $\lambda \mathbf{Z}_{1}+\lambda^{2} \mathbf{Z}_{2}+\lambda^{3} \mathbf{Z}_{3}+\ldots$ converges at $\lambda=1$, if the terms $\mathbf{b}_{i j}$ of the matrix $\Delta \mathbf{B}$ (and consequently $\mathbf{h}_{i j}$ ) are sufficiently small. Thus, the energy of dispersion forces

$\Delta W=\frac{\hbar}{2}\left(\operatorname{Sp}\left[\mathbf{B}^{1 / 2}\right]-\operatorname{Sp}\left[\mathbf{B}_{0}^{1 / 2}\right]\right)=\frac{\hbar}{2} \operatorname{Sp}\left[\mathbf{Z}_{2}\right]+\frac{\hbar}{2} \operatorname{Sp}\left[\mathbf{Z}_{3}\right]+\frac{\hbar}{2} \operatorname{Sp}\left[\mathbf{Z}_{4}\right]+\ldots$,

can be presented as a sum of pairwise, triple, quadruple, etc. interactions (the term $\operatorname{Sp}\left[Z_{1}\right]=\sum_{i} \operatorname{Sp}\left[\mathbf{h}_{i i}\right]$ is equal to 0 , since all diagonal blocks $\left.\mathbf{h}_{i i} \equiv \mathbf{0}\right)$.

The term

$\frac{\hbar}{2} \operatorname{Sp}\left[\mathbf{Z}_{2}\right]=\frac{\hbar}{2} \sum_{i} \operatorname{Sp}\left[-\mu_{i i} \sum_{k} \mathbf{h}_{i k} \mathbf{h}_{k i}\right]=\sum_{i} \sum_{<k}\left\{-\frac{\hbar}{2} \cdot \frac{\omega_{i}+\omega_{k}}{2 \omega_{i} \omega_{k}} \operatorname{Sp}\left[\mathbf{h}_{i k} \mathbf{h}_{k i}\right]\right\}$ is a sum of conventional London's energies $\Delta W_{i k}[10-13]$ of pairwise interactions: since $\operatorname{Sp}\left[\theta_{i k} \theta_{k i}\right]=6$,

$$
\Delta W_{i k}=-\frac{3 \hbar\left(\omega_{i}+\omega_{k}\right)}{2} \cdot\left(\gamma_{i k}\right)^{2}
$$

where

$$
\gamma_{i k}=\frac{\sqrt{\omega_{i} \omega_{k}}}{\omega_{i}+\omega_{k}} \cdot\left(\frac{\sqrt{\alpha_{i} \alpha_{k}}}{\left|\mathrm{r}_{i k}\right|^{3}}\right)
$$

is a convenient dimensionless parameter for the interaction of oscillators $i$ and $k$, and $\alpha_{i}=e^{2} /\left(m_{i} \omega_{i}^{2}\right)$ is the electronic polarizability of atom $i$.

The term

$$
\frac{\hbar}{2} \operatorname{Sp}\left[\mathbf{Z}_{3}\right]=\sum_{i} \sum_{<k} \sum_{<p}\left\{\frac{\hbar}{2} \cdot \frac{\omega_{i}+\omega_{k}+\omega_{p}}{\omega_{i} \omega_{k} \omega_{p}} \cdot \operatorname{Sp}\left[\mathbf{h}_{i k} \mathbf{h}_{k p} \mathbf{h}_{p i}\right]\right\}
$$

is the sum of the energies of triple interactions, $\Delta W_{i k p^{\prime}}$ which depends on the geometry of the triangles (Fig 1a) formed by the atoms involved $[6,8,11,13]$. Because $\operatorname{Sp}\left[\theta_{i k} \theta_{k p} \theta_{p i}\right]=3+9 \cos \phi_{k} \cdot \cos \phi_{p} \cdot \cos \phi_{i^{\prime}}$

$\Delta W_{i k p}=\frac{3 \hbar\left(\omega_{i}+\omega_{k}+\omega_{p}\right)}{2} \cdot \gamma_{i k} \gamma_{k p} \gamma_{p i} \cdot\left(1+3 \cos \varphi_{i} \cos \varphi_{k} \cos \varphi_{p}\right)$, where $i \neq k \neq p \neq i$.

Thus, a triple interaction can be both repulsive and attractive: attractive, when atoms $i, k, p$ stay nearly along a line; repulsive, when these atoms form an acute or right-angled triangle [6].

The quadruple interactions (contributing to the energy term $\frac{\hbar}{2} \mathrm{Sp}\left[\mathrm{Z}_{4}\right]$ can consist of not only four different atoms, but also three or two. Four-, three- and two-atom interactions contain terms like $\operatorname{Sp}\left[\mathbf{h}_{i k} \mathbf{h}_{k p} \mathbf{h}_{p q} \mathbf{h}_{q i}\right], S p\left[\mathbf{h}_{i k} \mathbf{h}_{k i^{-}}\right.$ $\left.\mathbf{h}_{i p} \mathbf{h}_{p i}\right], \operatorname{Sp}\left[\mathbf{h}_{i k} \mathbf{h}_{k i} \mathbf{h}_{i k} \mathbf{h}_{k i}\right]$, respectively (where different indices denote different particles). After simple but voluminous calculations it can be shown that

$$
\frac{\hbar}{2} \operatorname{Sp}\left[\mathbf{Z}_{4}\right]=\sum_{i} \sum_{<k} 2 \Gamma_{i k i k}+\sum_{i} \sum_{<k} \sum_{<p}\left(4 \Gamma_{i k i p}+4 \Gamma_{k i k p}+4 \Gamma_{p i p k}\right)+\sum_{i} \sum_{<k} \sum_{<p}\left(8 \Gamma_{i k p q}+8 \Gamma_{i k q p}+8 \Gamma_{i p k q}\right),
$$




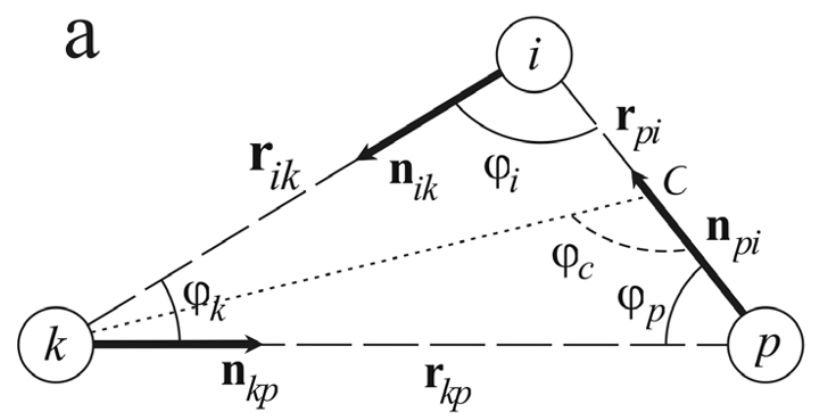

$\operatorname{Sp}\left[\boldsymbol{\theta}_{i k} \boldsymbol{\theta}_{k p} \boldsymbol{\theta}_{p i}\right]=3+9 \cos \varphi_{i} \cos \varphi_{k} \cos \varphi_{p}$

$$
\begin{gathered}
\text { if }\left|\mathbf{r}_{i k}\right| \gg\left|\mathbf{r}_{p i}\right|,\left|\mathbf{r}_{k p}\right| \gg\left|\mathbf{r}_{p i}\right|: \\
\Delta W_{i k p} \approx 9 \hbar \bar{\omega}_{\Delta} \gamma_{C k}^{2} \frac{1-3 \cos ^{2} \varphi_{C}}{2} \gamma
\end{gathered}
$$

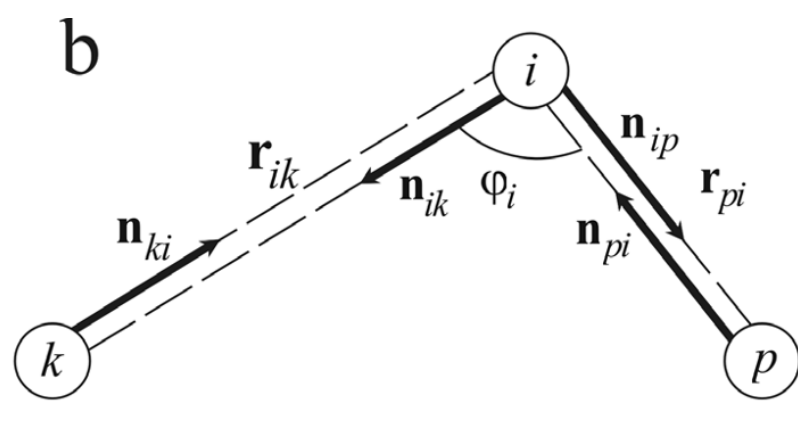

$-\mathrm{Sp}\left[\boldsymbol{\theta}_{i k} \boldsymbol{\theta}_{k i} \boldsymbol{\theta}_{i p} \boldsymbol{\theta}_{p i}\right]=-9-9 \cos ^{2} \varphi_{i}$

$$
\begin{aligned}
\Delta W_{i k i p}= & -15 \hbar \bar{\omega}_{\Leftrightarrow} \gamma_{i k}^{2} \gamma^{2} \\
& +15 \hbar \bar{\omega}_{\Leftrightarrow} \gamma_{i k}^{2} \frac{1-3 \cos ^{2} \varphi_{i}}{4} \gamma^{2}
\end{aligned}
$$

\section{Figure I}

Geometrical factors that determine (a) triple and (b) one out of three quadruple interactions of three atoms: $i, k, p$. $C$ is the center of the shortest $(i-p)$ side of triangle; $\gamma=\gamma_{p i} ; \bar{\omega}_{\Delta}=\left(\omega_{i}+\omega_{k}+\omega_{p}\right) / 3$;

$\bar{\omega}_{\Leftrightarrow}=\left[2 \omega_{i}+\omega_{k}+\omega_{p}+\omega_{k} \omega_{p} / 2 \omega_{i}+\omega_{i}^{2} /\left(\omega_{k}+\omega_{p}\right)\right] / 5$.

where

$\Gamma_{i k p q}=\frac{\hbar}{16}\left(\omega_{i}+\omega_{k}+\omega_{p}+\omega_{q}+\frac{\omega_{k} \omega_{q}}{\omega_{i}+\omega_{p}}+\frac{\omega_{i} \omega_{p}}{\omega_{k}+\omega_{q}}\right) \cdot \gamma_{i k} \gamma_{k p} \gamma_{p q} \gamma_{q i} \cdot\left(-\operatorname{Sp}\left[\boldsymbol{\theta}_{i k} \boldsymbol{\theta}_{k p} \boldsymbol{\theta}_{p q} \boldsymbol{\theta}_{q i}\right]\right)$

In two-atom quadruple terms $\Gamma_{i k i k^{\prime}}-\operatorname{Sp}\left[\theta_{i k} \theta_{k i} \theta_{i k} \theta_{k i}\right]=-18$. In three-atom quadruple terms like $\Gamma_{i k i p}$

$$
-\operatorname{Sp}\left[\theta_{i k} \theta_{k i} \theta_{i k} \theta_{p i}\right]=9-9 \cos ^{2} \phi_{i}
$$

is always negative (Fig. 1b), i.e., neighbor $k$ of atom $i$ increases attraction of $i$ to $p$, and neighbor $p$ of $i$ increases attraction of $i$ to $k$. In four-atom quadruple terms $\Gamma_{i k p q}$ the value of $-\operatorname{Sp}\left[\theta_{i k} \theta_{k p} \theta_{k p} \theta_{q i}\right]$ varies from -18 to +9 depending on the mutual arrangement all four atoms.

\section{Microscopic dielectric permittivity in a system of oscillators}

Consider the system of oscillators described above, where dipole moments $e \mathrm{x}_{a^{\prime}} e \mathrm{x}_{b}$ of atoms $a, b$ are now fixed. The potential energy of this system is

$$
U=\mathbf{x}_{a} \mathbf{g}_{a b} \mathbf{x}_{b}+\mathrm{X}^{(a b)} \mathbf{G}_{a}^{(a b)} \mathbf{x}_{a}+\mathrm{X}^{(a b)} \mathbf{G}_{b}^{(a b)} \mathbf{x}_{b}+1 / 2 \mathbf{X}^{(a b)} \mathbf{A}^{(a b)} \mathbf{X}^{(a b)}
$$

Here $\mathbf{g}_{a b}=e^{2} \theta_{a b} /\left|\mathrm{r}_{a b}\right|^{3}, \mathrm{X}^{(a b)}$ is a $3 \cdot(n-2)$-dimensional vector composed of $n-23 \mathrm{D}$ vectors $\mathrm{x}_{i}(i \neq a, b), \mathbf{G}_{a}^{(a b)}, \mathrm{G}_{b}^{(a b)}$ are $3 \times 3 \cdot(n-2)$ matrices composed of $n-23 \mathrm{D}$ matrices $\mathbf{g}_{i a}$ or $\mathrm{g}_{i b}$, respectively $(i \neq a, b)$, and the $3 \cdot(n-2) \times 3 \cdot(n-2)$ matrix $\mathbf{A}^{(a b)}=\left\|\mathbf{a}_{i j}\right\|$ is composed of $(n-2) \times(n-2) 3$ D blocks $\mathbf{a}_{i j}[i \neq$ $a, b, j \neq a, b, \mathbf{a}_{i j}=m_{i} \omega_{i}^{2} \delta$ if $i=j, \mathbf{a}_{i j}=\mathbf{g}_{i j}$ if $\left.i \neq j\right]$. The system's energy minimum is determined by equations $\frac{d}{d \mathrm{x}^{(\mathrm{ab})}} U=0, \quad$ so that $\mathrm{X}^{(a b)}=-\left[\mathbf{A}^{(a b)}\right]^{-1} \mathbf{G}_{a}^{(a b)} \mathbf{X}_{a}-\left[\mathbf{A}^{(a b)}\right]^{-1} \mathbf{G}_{b}^{(a b)} \mathbf{x}_{b}$, and the energy at minimum is

$U_{\min }=\left\{-1 / 2 \mathbf{x}_{a} \mathbf{G}_{a}^{(a b)}\left[\mathbf{A}^{(a b)}\right]^{-1} \mathbf{G}_{a}^{(a b)} \mathbf{x}_{a}-1 / 2 \mathbf{x}_{b} \mathbf{G}_{b}^{(a b)}\left[\mathbf{A}^{(a b)}\right]^{-1} \mathbf{G}_{b}^{(a b)} \mathbf{x}_{b}\right\}-\mathbf{x}_{a} \mathbf{G}_{a}^{(a b)}\left[\mathbf{A}^{(a b)}\right]^{-1} \mathbf{G}_{b}^{(a b)} \mathbf{x}_{b}+\mathbf{x}_{a} \mathbf{g}_{a b} \mathbf{x}_{b}$.

The terms in \{\} are not interesting to us at this stage, they correspond to the interaction of separate fixed dipoles $a$ and $b$ with a polarizable environment. The term $-\mathbf{x}_{a} \mathbf{G}_{a}^{(a b)}\left[\mathbf{A}^{(a b)}\right]^{-1} \mathbf{G}_{b}^{(a b)} \mathbf{x}_{b}$ corresponds to the interaction of dipoles $a$ and $b$ via a polarizable environment; and the last term corresponds to the direct interaction of dipoles $a$ 
and $b$. Thus, the polarizable medium-modified energy of electrostatic interaction of fixed dipoles $a$ and $b$ is

$$
U_{a \text {-medium- } b}^{(1)}=\mathbf{x}_{a}\left(\mathbf{g}_{a b}-\mathbf{G}_{a}^{(a b)}\left[\mathbf{A}^{(a b)}\right]^{-1} \mathbf{G}_{b}^{(a b)}\right) \mathbf{x}_{b}
$$

when the "medium" consists of oscillators described at a microscopic level.

On the other hand, we have a classic "macroscopic" equation for the medium-modified energy of interaction of the same two dipoles. The modification is described by permittivity, $\varepsilon$ :

$$
U_{a b}^{(\varepsilon)}=\mathrm{x}_{a}\left[\frac{1}{\varepsilon}\right] \mathbf{g}_{a b} \mathrm{x}_{b}
$$

The value $\frac{1}{\varepsilon}$ is a scalar in a uniform macroscopic medium; but $\left[\frac{1}{\varepsilon}\right]_{a b}=\boldsymbol{\delta}-\mathbf{G}_{a}^{(a b)}\left[\mathbf{A}^{(a b)}\right]^{-1} \mathbf{G}_{b}^{(a b)} \mathbf{g}_{a b}^{-1}$ is a $3 \times 3$ tensor that depends on the 3D positions of dipoles $a$ and $b$ when in a non-uniform "microscopic" medium. This can be seen from comparison of Equations [18] and [19], which describe the same energy $U^{(1)}{ }_{a \text {-medium- } b}=U^{(\varepsilon)}{ }_{a b}$ in "micro-" and "macroscopic" terms. However, the effective value of medium-induced modification of dipole-dipole interaction strength can still be defined by the equation

$$
\frac{1}{\varepsilon_{a b}^{2}}=\operatorname{Sp}\left[\left[\frac{1}{\varepsilon}\right]_{a b} \mathbf{g}_{a b}\left[\frac{1}{\varepsilon}\right]_{b a} \mathbf{g}_{b a}\right] / \operatorname{Sp}\left[\mathbf{g}_{a b} \mathbf{g}_{b a}\right] .
$$

Defined thus, the scalar $\varepsilon_{a b}$ coincides with the conventional permittivity in a uniform macroscopic medium. When the "medium" consists of oscillators described at a microscopic level, $\varepsilon_{a b}$ corresponds to the dielectric permittivity for the interaction of dipoles $a$ and $b$ via the polarizable environment.

From equations [18] - [20],

$$
\varepsilon_{a b}^{-2}=\operatorname{Sp}\left[\left(\mathbf{g}_{a b}-\mathbf{G}_{a}^{(a b)}\left[\mathbf{A}^{(a b)}\right]^{-1} \mathbf{G}_{b}^{(a b)}\right)\left(\mathbf{g}_{b a}-\mathbf{G}_{b}^{(a b)}\left[\mathbf{A}^{(a b)}\right]^{-1} \mathbf{G}_{a}^{(a b)}\right)\right] / \operatorname{sp}\left[\mathbf{g}_{a b} \mathbf{g}_{b a}\right]
$$

. To estimate this value with accuracy up to triple interactions (i.e. up to the third powers of small $\mathbf{g}$ matrices), it remains only to find the main (g-independent) term of $\left[\mathbf{A}^{(a b)}\right]^{-1}$, since products like $\mathbf{G}_{a}^{(a b)} \mathbf{G}_{b}^{(a b)}$ already contain second powers of $\mathbf{g}$. From expansion similar to that presented in equation [5], one obtains $\frac{1}{\varepsilon_{a b}^{2}} \approx 1-\sum_{p \neq a, b} \frac{2}{m_{p} \omega_{p}^{2}} \cdot \frac{\operatorname{Sp}\left[g_{a b} g_{b p} g_{p a}\right]}{\operatorname{Sp}\left[g_{a b} g_{b a}\right]}=1-\sum_{p \neq a, b} \frac{2 \alpha_{p}\left|\mathrm{r}_{a b}\right|^{3}}{\left|\mathrm{r}_{a p}\right|^{3}\left|\mathrm{r}_{p b}\right|^{\beta}} \cdot \frac{\operatorname{Sp}\left[\boldsymbol{\theta}_{a b} \boldsymbol{\theta}_{b p} \boldsymbol{\theta}_{p a}\right]}{\operatorname{Sp}\left[\boldsymbol{\theta}_{a b} \boldsymbol{\theta}_{b a}\right]}$.

If the sum is taken over the oscillating electrons only (while the nuclei positions are fixed), $\varepsilon_{a b}$ is an electronic component of dielectric permittivity.

\section{Multi-atom VdW interactions are important in the presence of covalent bonds}

Equations [10] - [14] show that energies of pairwise, triple and quadruple interactions are proportional to $\sim \hbar \omega \cdot \gamma_{i k} \gamma_{k i^{\prime}} \sim \hbar \omega \cdot \gamma_{i k} \gamma_{k p} \gamma_{p i}$ and $\sim \hbar \omega \cdot \gamma_{i k} \gamma_{k p} \gamma_{p q} \gamma_{q i^{\prime}}$ respectively, where $\hbar \omega$ is an oscillator excitation energy. The $\gamma$ values are small for non-bonded contacts. Indeed, $\frac{\sqrt{\omega_{i} \omega_{k}}}{\omega_{i}+\omega_{k}}$ in $\gamma_{i k}=\frac{\sqrt{\omega_{i} \omega_{k}}}{\omega_{i}+\omega_{k}} \cdot\left(\frac{\sqrt{\alpha_{i} \alpha_{k}}}{\left|\mathrm{r}_{i k}\right|^{3}}\right)$ is normally close to $1 / 2$, because $\omega_{i}$ and $\omega_{k}$ are of equal order of magnitude [13], and the $\alpha$ values are about $1 \AA^{3}$ for atoms typical in proteins $\left(0.40 \AA^{3}\right.$ for $\mathrm{H}$ atoms, $0.69-0.85 \AA^{3}$ for $\mathrm{O}$ atoms, 0.90-0.97 $\AA^{3}$ for $\mathrm{N}$ atoms, $1.03-1.32 \AA^{3}$ for $\mathrm{C}$ atoms [13]). Thus, the dimension less value $\gamma$ is about 0.02 for the closest non-bonded contact of atoms $(|r| \approx 2.2-3.4 \AA$ for atoms $\mathrm{H}, \mathrm{O}, \mathrm{N}, \mathrm{C}[13])$.

The very small value of $\gamma$ at "non-bonded" distances means that triple, quadruple, etc., non-bonded atomatom contacts are very weak compared to those that are pairwise.

However, the situation changes when the $\gamma$ factor involves two atoms connected by a covalent bond. The atom-atom distance in this case is at least two times smaller than that for the closest non-bonded contact. Thus, the $\gamma$ factor for covalently bonded atoms is about tenfold (see Eq. [11]) larger than the $\gamma$ factor for the closest non-bonded contact of atoms. In this case, the energy of high-order interactions can approach that of a pairwise interaction.

Taking an average estimate of $\gamma=0.15$ for bonded atoms (that corresponds to a typical covalent bond length of 1.5 $\AA$ A between chemically equal atoms with polarizability of $1 \AA^{3}$ ), we see (Fig. 2) a significant change in the VdW interaction energy for the cost of triple and quadruple interactions of three atoms (when a pair of these atoms is covalently bonded) and, to a lesser extent, for the cost of four-atom interactions (when two pairs of atoms are covalently bonded). It is useful to mention here that one study cited herein [14] has already discussed the general idea that "bond-bond" interactions are more appropriate 


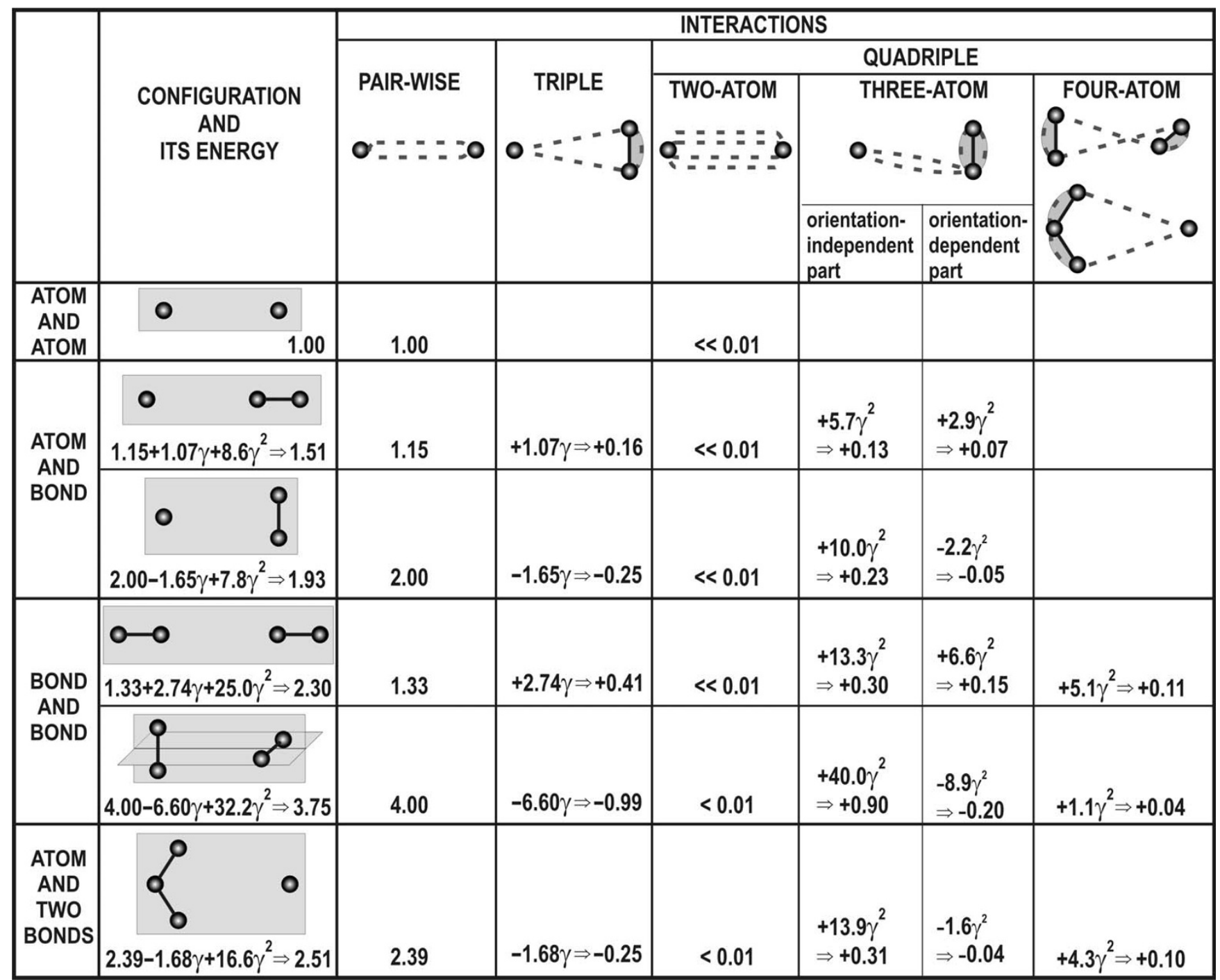

Figure 2

Energy of VdW interactions at various configurations of participating non-bonded and bonded atoms. The distance between the closest non-bonded atoms is $4 \AA$ in all cases, and unity is used as the energy of pairwise interaction of atoms at $4 \AA$ distance. If the distance between the closest non-bonded atoms is $|r| \approx 4 \AA$, all energies presented in the Table change as $(4 \AA /|r|)^{6}$, approximately, and their ratio does not change essentially. The value $\gamma=0.15$ describes the coupling of oscillations in two atoms connected by a covalent bond of fixed length I.5 A (see text for further explanation).

for conformational analysis than "atom-atom" interactions.

In the case of the collinear arrangement of atoms and bonds the attraction can grow by $70 \%$ (compare values in columns "configuration and its energy" and "pairwise" in Fig. 2), while in the case of the orthogonal arrangement of atoms and bonds, the attraction can decrease by $6 \%$. The decrease is less than the increase because, as mentioned above, the quadruple interaction is mainly attractive. The main part of this attraction is due to the three-atom quadruple interaction. This can be divided in two parts: Equa- tion [15] can be presented as $\operatorname{Sp}\left[\theta_{i k} \theta_{k i} \theta_{i p} \theta_{p i}\right]=12+\left(9 \cos ^{2} \phi_{i}\right.$ - 3), where the first (larger) term is an orientation-independent constant (actually, it simply increases the VdW attraction of any atom involved in covalent bonding to all other atoms, see Fig. 1b; in available force fields [1-5] this term is implicitly taken into account by ascribing and fitting different pairwise attraction forces to atoms in different covalent states). The second, the orientationdependent part equals zero if averaged in $3 \mathrm{D}$ space over all possible atom-to-bond orientations (see examples in Fig. 2); this part is rather similar to that for the interaction 
of a separate atom with a remote covalent bond (cf. Fig.1b to Fig. 1a).

The orientation-dependent terms, which mainly arise from triple interactions, can increase or decrease $\mathrm{VdW}$ energy by $\sim 20-40 \%$ at extremes. This energy effect is significant, being comparable to that caused by the replacement of an $\mathrm{N}$ atom $\left(\alpha_{N} \approx 0.95 \AA^{3}\right)$ by a C $\left(\alpha_{C} \approx 1.2 \AA^{3}\right)$ or $\mathrm{O}\left(\alpha_{\mathrm{O}} \approx 0.75 \AA^{3}\right)$ atom in a pairwise VdW interaction (see equations $[10,11]$ at $\left.\omega_{N} \approx \omega_{C} \approx \omega_{O}\right)$. Moreover, the orientation effect is much stronger than the effect of replacing the pairwise energies of two C-N non-bonded contacts with the sum of pairwise energies of $\mathrm{C}-\mathrm{C}$ and $\mathrm{N}-\mathrm{N}$ contacts $\left(\alpha_{C} \alpha_{C}+\alpha_{N} \alpha_{N}-2 \alpha_{C} \alpha_{N} \approx 5 \% \times \alpha_{C} \alpha_{N}\right)$. Note that the contact replacements are the main VdW effects currently used to distinguish "correct" protein folds from those that are "wrong".

Thus, we indeed see that any conformational analysis should by no means ignore the cumulative effect of the coupling of multi-atom VdW interactions with covalent bonds. Figure 3 shows that the energy difference between two structures of equal compactness may change twice due to this effect.
It is necessary to note that at short distances, which are the most important for $\mathrm{VdW}$ interactions, one can expect effects of inharmonicity, higher multipole interactions, etc. $[11,12]$. These effects are avoided in the "harmonic oscillator" model used in this work. Nevertheless, they exist in reality, and their existence makes the expressions obtained for many-body interactions approximate to the same extent as the conventional London's expression is for pairwise interactions. In particular, the value of $\gamma$ for each covalent bond may be treated as a parameter, the value of which should be obtained from a fit of the experimental data in the same way as the London's pairwise interaction energy $[11,13,14]$. The necessity of fitting the experimental data is underlined by a relatively slow convergence of both the orientation-dependent and orientation-independent series in Figure 2.

\section{"VdW permittivity"}

VdW forces and the electronic part of dielectric permittivity are evidently connected: both originate from dipoledipole interactions. The relationship between VdW forces and the macroscopic dielectric permittivity of the medium is well studied in the continuum approximation $[11,14,16]$. However, this approach is useful and rela-

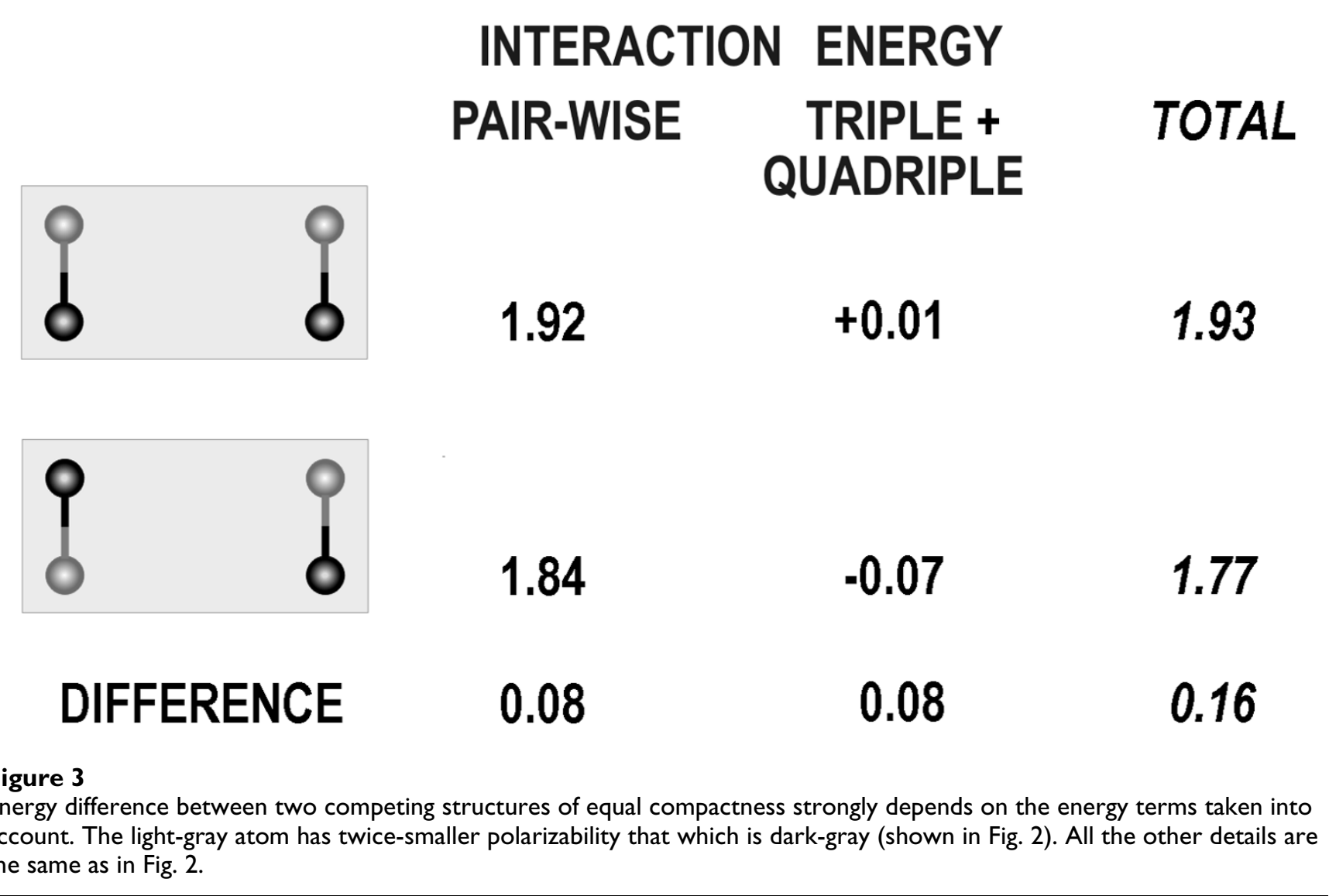


tively simple when applied to large uniform bodies, like drops or layers. This work concerns interactions of small fragments composed of various atoms, and the frequencydependent dielectric permeability, taken as a macroscopic characteristic, seems not to be an appropriate tool to investigate this case. Here we will consider an electronic part of the microscopic dielectric permittivity created by and acting at interacting harmonic oscillators, with the goal of demonstrating that multi-atom interaction creates a kind of "VdW permittivity" for pairwise VdW forces in addition to elucidating a connection between VdW forces and the electronic part of microscopic dielectric permittivity.

The role of dielectric permittivity in VdW forces is ambiguous. On the one hand, the VdW interaction of two atoms is proportional to the square of the electrostatic interaction between their fluctuating electronic polarizations. Thus, one might expect the pairwise VdW interaction to be inversely proportional to the square of the electronic part of the dielectric permittivity. On the other hand, the medium's electrons (which create the electronic component of dielectric permittivity) are involved in VdW interactions of "their own" atoms, and it is not clear if they are "free" enough to influence the VdW interactions of the other atoms in such a strong manner.

Following equations [8] - [10], [12], [13], one can present the total VdW energy $\Delta W$ as

$$
\begin{gathered}
\sum_{i} \sum_{<k} \Delta W_{i k}+\sum_{i} \sum_{<k} \sum_{<p} \Delta W_{i k p}+\ldots= \\
\sum_{i<k} \sum_{<k} \Delta W_{i k}\left\{1-\sum_{p \neq i, j} \frac{\left(\omega_{p}\right)^{2}}{\left(\omega_{i}+\omega_{p}\right)\left(\omega_{p}+\omega_{k}\right)} \cdot \frac{2 \alpha_{p}\left|\mathrm{r}_{i k}\right|^{3}}{\left|\mathrm{r}_{i p}\right|^{3}\left|\mathrm{r}_{p k}\right|^{3}} \cdot \frac{\operatorname{Sp}\left[\boldsymbol{\theta}_{i k} \boldsymbol{\theta}_{k p} \boldsymbol{\theta}_{p i}\right]}{\operatorname{Sp}\left[\boldsymbol{\theta}_{i k} \boldsymbol{\theta}_{k i}\right]}+\ldots\right\}
\end{gathered}
$$

and

see

that

$\Phi_{i k} \approx\left\{1-\sum_{p \neq i, j} \frac{\left(\omega_{p}\right)^{2}}{\left(\omega_{i}+\omega_{p}\right)\left(\omega_{p}+\omega_{k}\right)} \cdot \frac{2 \alpha_{p}\left|\mathrm{r}_{i k}\right|^{3}}{\left|\mathrm{r}_{i p}\right|^{3}\left|\mathrm{r}_{p k}\right|^{3}} \cdot \frac{\operatorname{Sp}\left[\boldsymbol{\theta}_{i k} \boldsymbol{\theta}_{k p} \boldsymbol{\theta}_{p i}\right]}{\operatorname{Sp}\left[\boldsymbol{\theta}_{i k} \boldsymbol{\theta}_{k i}\right]}+\ldots\right\}^{-1}$ plays the role of "microscopic permittivity" for the pairwise VdW interactions of atoms $i, k$. This "permittivity" depends on the distance between atoms $i$ and $k$, and the space distribution of the other atoms with oscillating electrons around them. The value $\Phi_{i k}$ looks similar to, but does not coincide with the square of the electronic part of dielectric permittivity $\varepsilon_{a b}^{2}$ given by equation [21]. Thus the naive idea that VdW interaction may be inversely proportional to the square of the electronic part of the dielectric permittivity turned out to be wrong. However, the values $\Phi_{i k}$ and $\varepsilon_{i k}$ are related if all atoms have equal electron oscillation frequencies $\omega$. Since the value $\varepsilon_{i k}$ of the electronic component of the dielectric permittivity is not far from unity [13], we see in this case that

$$
\Phi_{i k} \approx \sqrt{\varepsilon_{i k}} .
$$

Thus, on average, MB effects decrease the pairwise VdW energy roughly in proportion to the square root of the electronic part of the microscopic dielectric permittivity for the dipole-dipole interaction via a polarizable atomic environment.

The main effect is caused by the electronic permittivity, which is pertinent to small, atomic distances, where the main VdW interaction takes place. One can expect that the electronic component of the microscopic dielectric permittivity for interacting dipoles at these distances is significantly smaller than the electronic dielectric permittivity at macroscopic distances: the decrease in VdW energy caused by three-atom interaction in liquid argon [7] is approximately five-fold less than that expected the from its macroscopic permittivity.

\section{Conclusion}

Ligand binding and protein structure prediction require especially accurate force field potentials, because one "correct" structure struggles against billions of "wrong" ones [17]. Despite huge efforts to optimize them, the force fields currently used are still not able, in a vast majority of cases, to bring an approximate, homology-based model of protein structure (whose atoms usually deviate by only 1.5 - $3 \AA$ from their native positions) closer to its native conformation [18-22]. At present, even the most successful methods (one or two of the five dozen methods used) show some improvement of homology models by a force field-based refinement in only a half of cases [21-23]. This shows that a "significant difficulty still exists in both sampling and force field accuracy" [23]. One of the force field problems is that which concerns the "not well captured" balance between intramolecular and solvent dispersion (i.e., VdW) interactions [23]. On the other hand, a partial success of modern molecular mechanics methods in the prediction of detailed protein structure shows that (i) modern, improved methods of sampling of protein chain conformations seem to work (possibly, at the cost of averaging that decreases the effect of energy errors [17]) in some, but not all, cases (especially when a near-native conformation basin is reached from homology modeling), and (ii) current force field quality is sufficient in some, but not all, cases, so that an increase in the accuracy of the force field seems to be crucial for the final success of protein structure prediction methods.

This study shows that currently-ignored multi-atom VdW interactions can be important for the further development of force fields. The coupling of covalent bonding with 
multi-atom VdW interactions is not the only important $\mathrm{MB}$ effect. Some of those, like MB electrostatic interactions, are taken into account by the existing force fields (see $[24,25])$. Here the effect is caused by the interaction of the permanent charges or permanent molecular multipoles with particles of polarizable media. The other interactions, which include interaction of permanent multipoles with induced ones [26], are not yet taken into account by existing force fields.

The discovered specific coupling of covalent bonding with multi-atom VdW interactions does not need permanent charges or multipoles, and therefore involves the multitude of covalent bonds in molecules. Multi-atom interaction with any of the bonds can lead to a significant energy effect, which is comparable to that caused by the replacement of atoms in conventional pairwise $\mathrm{VdW}$ interactions. As a result, the effect described can be rather significant in choosing the molecular shape.

\section{Abbreviations}

3D - three-dimensional; VdW - Van der Waals; MB multi-body; CASP - Critical Assessment of Techniques for Protein Structure Prediction meeting

\section{Acknowledgements}

I am grateful to D. Reifsnyder for assistance in the preparation of this paper, to A.G. Donchev for assistance in computations and to A.M. Dykhne, A.A. Vedenov, R.V. Polozov, M. Levitt and G. Vriend for seminal discussions. This work was supported in part by the Russian Foundation for Basic Research and Russian programs "Molecular and Chemical Biology" and "Leading Scientific Schools", INTAS, IHES and an International Research Scholar's Award from the Howard Hughes Medical Institute.

\section{References}

I. Levitt M, Hirshberg M, Sharon R, Dagget V: Potential energy function and parameters for simulations of the molecular dynamics of proteins and nucleic acids in solution. Comp Phys Commun 1995, 91:215-231.

2. MacKerell A Jr, Wiorkiewicz-Kuczera J, Karplus M: An all-atom empirical energy function for the simulation of nucleic acids. J Am Chem Soc 1995, I I 7: I 1946-I I 975.

3. Jorgensen WL, Maxwell DS, Tirado-Rives J: Development and testing of the opls all-atom force field on conformational energetics and properties of organic liquids. J Am Chem Soc 1996, II 8: II 225-11236

4. Halgren TA: Merck molecular force field. I. Basis, form, scope, parameterization and performance of MMFF94. J Comp Chem $1995,17: 490-519$

5. Wang J, Wolf RM, Caldwell JW, Kollman PA, Case DA: Development and testing of a general amber force field. I Comp Chem 2004, 25: II57-II74.

6. Axilrod BM, Teller E: Interaction of the van der Waals' type between three atoms. J Chem Phys 1943, I I:299-300.

7. Sadus RJ: Exact calculation of the effect of three-body AxilrodTeller interactions on vapour-liquid phase coexistence. Fluid Phase Equilibria 1998, | 44:351-360.

8. MacRury TB, Linder B: Many-body aspects of physical adsorption. J Chem Phys 1971, 54:2056-2966.

9. Donchev AG: Many-body effects of dispersion interaction. I Chem Phys 2006, 125:0747|13.

10. London F: The general theory of molecular forces. Trans Faraday Soc 1937, 33:8-26.
II. Barash YS: Van der Waals Forces Volume chapters 2, 5. Moscow: Nauka; 1988.

12. Landau LD, Lifshitz EM: Quantum Mechanics Volume §23. New York: Pergamon; 1987.

13. Pauling L: General chemistry Volume chapters 6, II. New York: W.H.Freeman \& Co; 1970.

14. Parsegian AV: VdW Forces Cambridge: Cambridge Univ. Press; 2005. pp.4-29, 2I 4-227, 24I-260

15. Basharov MA, Vol'kenshtein MV, Golovanov IB, Ermakov GL, Nauchitel' VV, Sobolev VM: Bond-bond interactions. I. Simple relationship for estimating the energy of a bond-bond interaction. I Struct Chem (Moscow) 1984, 25:26-35.

16. Dzyaloshinskii IE, Lifshitz EM, Pitaevskii LP: General theory of Van der Waals' forces. Sov Phys Uspekhi I96I, 4:153-176.

17. Finkelstein AV, Gutin AM, Badretdinov AY: Perfect temperature for protein structure prediction and folding. Proteins 1995, 23:151-162.

18. Krieger E, Darden T, Nabuurs SB, Finkelstein A, Vriend G: Making optimal use of empirical energy functions: force field parameterization in crystal space. Proteins 2004, 57:678-683.

19. Vincent JJ, Tai C-H, Sathyanarayana BK, Lee B: Assessment of CASP6 predictions for new and nearly new fold targets. Proteins 2005:67-83.

20. Clarke N: Free modeling assessment. 2006 [http://prediction center.gc.ucdavis.edu/casp $7 /$ meeting/ presentationPresentations assessors/CASP7 FM Clarke.pdf].

21. Read RJ: Assessing high accuracy models. 2006 [http://predic tioncenter.gc.ucdavis.edu/casp $7 /$ meeting/ presentationPresentations assessors/CASP7 HA RJRead.pdf.

22. Schwede T: Assessment of template based modeling (TMB). 2006 [http://predictioncenter.gc.ucdavis.edu/casp7/meeting/presenta tions/Presentations assessors/CASP7 TBM Schwede.pdf].

23. Chen J, Brooks CL III: Can molecular dynamics simulations provide high-resolution refinement of protein structure? Proteins 2007, 67:922-930.

24. Honig B, Nicholls $A$ : Classical electrostatics in biology and chemistry. Science 1995, 268: | |44-I | 49.

25. Simonson T, Archontis G, Karplus M: Free energy simulations come of age: protein-ligand recognition. Acc Chem Res 2002, 35:430-437.

26. Ernesti A, Hutson JM: Nonadditive intermolecular forces from spectroscopy of van der Waals trimers: A theoretical study of $\mathrm{Ar}_{2}$-HF. Phys Rev A 1995, 5 I:239-250.

\section{Publish with ChemistryCentral and every scientist can read your work free of charge \\ "Open access provides opportunities to our \\ colleagues in other parts of the globe, by allowing \\ anyone to view the content free of charge." \\ W. Jeffery Hurst, The Hershey Company. \\ - available free of charge to the entire scientific community \\ - peer reviewed and published immediately upon acceptance \\ - cited in PubMed and archived on PubMed Central \\ - yours - you keep the copyright \\ Submit your manuscript here: \\ http://www.chemistrycentral.com/manuscript/

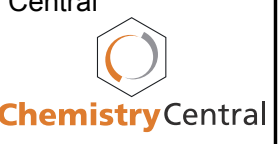

